\title{
Measurement accuracy of the pulse repetition interval-based excess fraction (PRIEF) method: an analogy-based theoretical analysis
}

D. Wei

weidong@nanolab.t.u-tokyo.ac.jp

\section{H. Matsumoto}

\begin{abstract}
Global Centers of Excellence Program for Mechanical Systems Innovation, School of Engineering, The University of Tokyo, Hongo 7-3-1, Bunkyo, Tokyo 113-8656, Japan

Department of Precision Engineering, The University of Tokyo, Hongo 7-3-1,Bunkyo-ku, Tokyo 1138656, Japan
\end{abstract}

We describe a novel approach for a theoretical analysis of the measurement accuracy of the pulse repetition interval-based excess fraction (PRIEF) method, which is expected to be useful for high-precision length measurement. The proposed approach is introduced by focusing on an analogy between the PRIEF method and the conventional length-measurement method. The theoretical analysis results show that the absolute accuracy achievable by the PRIEF method is nanometer-order, and the relative accuracy achievable by the PRIEF method is $10^{-8}$-order, which is affected mainly by the measuring accuracy of the refractive index of air. We conclude that our analysis is useful for further development of the PRIEF method.

[DOI: http://dx.doi.org/10.2971/jeos.2012.12050]

Keywords: Instrumentation, measurement mode-locked lasers, interferometry, metrology

\section{INTRODUCTION}

In 2009, a femtosecond optical frequency comb (FOFC) was adopted in Japan as the national standard tool for measuring length. Because of its stable frequency performance, the FOFC is believed to have brought about fundamental improvements in the accuracy of length measurements [1]-[3] However, the FOFC has not reached its full potential, since there is as yet no easy-to-use standard technology directly linked to the FOFC length standard tool for performing distance measurements (at the time of this publication); this has limited the development and applications of FOFC-based length measurement. (for example, see [4]-[6]).

To challenge this problem, we proposed the pulse repetition interval-based excess fraction (PRIEF) method [7]-[9], which can be realized in a modified Michelson interferometer for an arbitrary and absolute length measurement. We also reported a single pulse repetition interval version of PRIEF method, that compared to the conventional time-of-flight method - because interference fringes were used as the time-recording device-the PRIEF method can be expected to provide higher measurement accuracy [10].

In our previous report, [10] we obtained a 1.5-meter length measurement with a precision of several micrometers. The feasibility of the proposed method (though it was a single pulse repetition interval version PRIEF method) has thus been demonstrated. However, the expected high measurement accuracy was not achieved, due to the limited calibration accuracy of the gauge blocks we used and an inadequate experiment environment. In addition, we did not provide a theoretical estimation of the measurement limits of the PRIEF method, because of the journal's length-limit for reports.
To further test the performance of the PRIEF method, in the present study we split the accuracy influence factor into two parts based on the principles of the PRIEF method: the integer part and the fractional part. For the determination of the measurement uncertainties of these two parts, we introduce a new theoretical analysis approach. This approach is based on an analogy between the proposed method and the conventional length-measurement method. The error of each of the two parts is estimated in comparison with those of conventional methods whose measurement errors are well established.

Here, we give explanations in order to show the relation of the PRIEF method to length measurement standard FOFC tool. PRIEF method can be used to practically perform a distance metrology that is directly linked to an FOFC length standard tool. Just as a conventional Excess Fraction method can determine an arbitrary and absolute length of a gauge block based on the wavelength of a monochromatic laser source (for example, an iodine-stabilized helium-neon laser, which is the former national standard tool for measuring length), the PRIEF method can range an arbitrary and absolute length as a function of the pulse repetition interval length of an FOFC.

The measurement principles of the PRIEF method are reviewed and summarized in Section 2. A theoretical evaluation of the measurement uncertainties of the PRIEF method is given in Section 3. This theoretical evaluation also explains the superiority of the PRIEF method. Our main conclusions and a discussion of future work are provided in Section 4. 


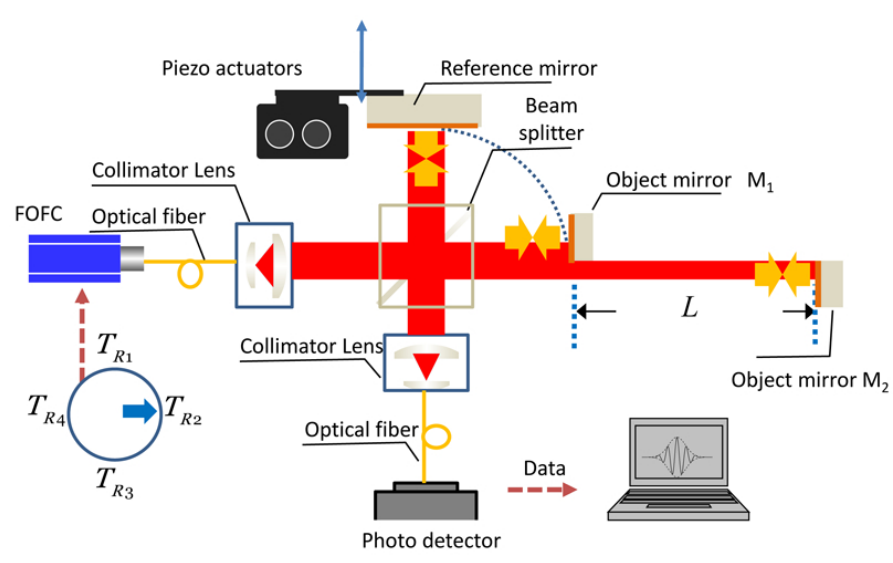

FIG. 1 Schematic diagram of a MPTI-based interferometer.

\section{PRINCIPLES OF THE PRIEF METHOD}

The PRIEF method can be regarded as a multi-pulse repetition interval version of the multiple pulse train interference-based (MPTI-based) length measurement method, about which a recent theoretical analysis [11] and experimental demonstration [10] were reported. For convenience of explanation, let us first briefly review the idea of obtaining an arbitrary and absolute length measurement with a modified Michelson interferometer, as illustrated in Figure 1.

A pulse train from an FOFC is introduced into a double Michelson interferometer. The double interferometer consists of two Michelson interferometers; one is an ordinary Michelson interferometer, and the other is an unbalanced opticalpath Michelson interferometer. The two Michelson interferometers consist of an identical beam splitter and a reference mirror, and different object mirrors (object mirror $\mathrm{M}_{1}$ and object mirror $\mathrm{M}_{2}$ ). Object mirror $\mathrm{M}_{2}$ is located far from object mirror $\mathrm{M}_{1}$, at an arbitrary length $L$. During the measurement, by moving the common reference mirror, we can observe the interference fringes for determining the length $L$.

The observed interference fringes can be written as: [11]

$$
\begin{aligned}
I(l) \propto & 2 \sqrt{I_{r e f} I_{o b j 1}} \times \exp \left[-\left(2 \sqrt{\ln 2} l / L_{c o h}\right)^{2}\right] \\
& \times \cos (k \times l)+2 \sqrt{I_{r e f} I_{o b j 2}} \\
& \times \exp \left[-\left(2 \sqrt{\ln 2}(l+\Delta) / L_{c o h}\right)^{2}\right] \\
& \times \cos \left(k \times(l+\Delta)-N \times \Delta \phi_{c e}\right) .
\end{aligned}
$$

where $I_{\text {ref, }} I_{o b j 1}$, and $I_{o b j 2}$ are the intensities reflected by the reference mirror, object mirror $\mathrm{M}_{1}$, and object mirror $M_{2}$, respectively. Value $T_{R}$ is the pulse repetition period of the FOFC source. When the electric field packet repeats at the pulse repetition period $T_{R}$, the "carrier" phase slips by $\Delta \phi_{c e}$ to the carrier-envelope phase because of the difference between the group and phase velocities. Value $l$ is the distance between the reference mirror and object mirror $M_{1}$. The equation $\Delta=\varepsilon \times c \times T_{R}=\bmod \left(2 L, N \times c \times T_{R}\right)$, $0 \leq \varepsilon<1$, returns $2 L-N \times c \times T_{R}$, the equation $N=$ floor $\left[2 L /\left(c \times T_{R}\right)\right]\left(\right.$ floor $\left[2 L /\left(c \times T_{R}\right)\right]$ rounds the elements of $2 L /\left(c \times T_{R}\right)$ to the nearest integers less than or equal to $2 l /\left(c \times T_{R}\right)$, and $c$ is the light velocity in vacuum. In addition, we assume that the FOFC light source used shows a Gaussian spectral distribution, and $L_{c o h}$ is the temporal coherence length of one pulse.

Because there is no need to move the object mirrors, we can understand that the length is measured in absolute value. If $\varepsilon$ and $N$ can be measured by any means, then an arbitrary and absolute distance $L$ can be decided as follows:

$$
L=(N+\varepsilon) \times c \times T_{R} / 2 .
$$

Eq. (2) means that if a pulse repetition interval $c \times T_{R}$ of an FOFC has sufficient stability as a ruler, an arbitrary and absolute distance $L$ can be estimation as a function of the pulse repetition interval $c \times T_{R}$. As described below, we consider the qualification of a pulse repetition interval $c \times T_{R}$ as a scale.

First, we consider the pulse repetition period $T_{R}$. The pulse repetition period $T_{R}$ and the pulse repetition frequency $f_{\text {rep }}$ are connected by $T_{R}=1 / f_{\text {rep }}$. Based on the theory of uncertainty propagation, we get $\sigma^{2}\left(\Delta T_{R} / T_{R}\right)=\sigma^{2}\left(\Delta f_{\text {rep }} / f_{\text {rep }}\right)$. Then, we turn our attention to the frequency domain for getting an estimate for $\sigma^{2}\left(\Delta f_{r e p} / f_{r e p}\right)$.

Currently, the highest absolute frequency stability that can be achieved by an FOFC is about $10^{-18}$-order [12]. In general, higher harmonics of the pulse repetition frequency is phaselocked to an radio frequency (RF) standard [13]. An RF standard can already provide frequency stability at the $10^{-13}$ and $10^{-14}$ levels $[14,15]$. The pulse repetition frequency can be simply detected by a photodiode. We can conclude that the measurement uncertainty of the pulse repetition frequency $\sigma^{2}\left(\Delta f_{\text {rep }} / f_{\text {rep }}\right)$ is higher than the $10^{-13}$ level. This means that the measurement uncertainty of the pulse repetition period is also higher than the $10^{-13}$ level.

Having analyzed the stability of the pulse repetition period $T_{R}$, we now turn to the topic of the measurement accuracy of the speed of light. The existence or absence of the propagation medium will affect the results of this analysis. In a vacuum, the speed of light is invariant. The speed of light in the vacuum cis defined as 299.792458 meters per second. Obviously, the accuracy is of the order $10^{-9}$.

Because length measurements are generally made in air, the propagation medium discussed here is limited to air. The discussion of other media can be done in a similar manner. In general, the Edlén Equation [16, 17] and Ciddor Equation [18] are used to obtain the refractive index of air $n$. Then the speed of light in air can be calculated as $c_{n}=c / n$. It is well known that the uncertainty of the Edlén Equation and Ciddor Equation is about $10^{-8}$-order.

From the above-mentioned analysis, we can conclude that the pulse repetition interval of an FOFC is very steady, and it is suitable as a ruler in vacuum and air. Thus far we have proven the utility of pulse repetition interval as a ruler for distance measurement.

As seen in Eq. (2), to measure length by using the pulse repetition interval of an FOFC, we must decide the integer part $N$ and the fraction part $\varepsilon$. As expressed in Eq. (1) and as shown in 


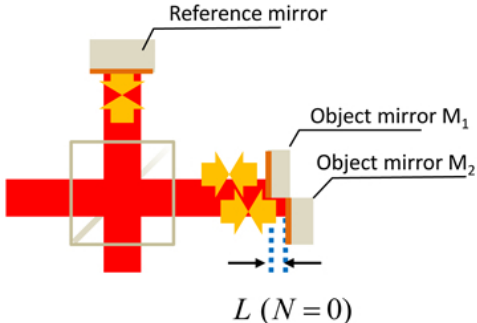

(a)

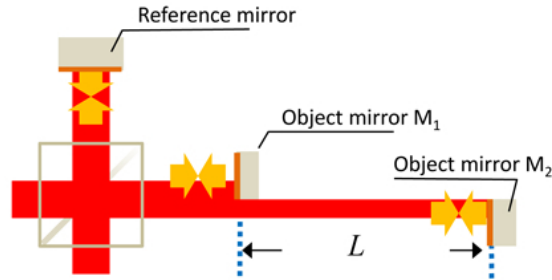

(b)

FIG. 2 Comparison of the white light method and the proposed method. (a) White light method. (b) Proposed mehtod.

our previous experiment, [10] we can measure a fraction part $\varepsilon$ by determining the distance between the peaks of obtained multiple pulse trains interference fringes. Regarding the integer part, we think that the use of the conventional Excess Fraction method $[19,20]$ will be appropriate, and this will be introduced later in the "uncertainties evaluation" section.

In our previous study, [11] we proposed the use of fringes analysis to measure a relative phase difference for obtaining an integer part. However, in air, phase is susceptible to atmospheric turbulence. In addition, the demand for the carrier phase slips, which is given by $\Delta \phi_{c e}=2 \pi f_{C E O} / f_{\text {rep }}$, is severe in air and vacuum. Because $\cos (\phi)=\cos (2 \pi+\phi)$, for determining the value of an integer part without $2 \pi$-ambiguity, $N \times \Delta \phi_{c e}<2 \pi$ is required. For a long-range measurement, the value of the integer part $N$ is large. That means that the carrier phase slips must be small enough, and the value of the offset shift frequency $f_{C E O}$ must be controlled to a value smaller than $f_{C E O}<f_{\text {rep }} / N$.

Before starting the error analysis, a summary of the principles of the PRIEF method is appropriate. First, pulse repetition interval is steady enough to serve as a ruler. Second, when a pulse repetition interval is used for an arbitrary and absolute length measurement, we need to determine an integer part and a fraction part. Lastly, we propose the use of the conventional Excess Fraction method to determine the integer part.

\section{UNCERTAINTIES EVALUATION BASED ON ANALOGY}

Here we consider the accuracy influence factor on the integer part and fractional part. For the uncertainties evaluation of the fraction part, we need to consider a special case, $N=0$. That means $L=\Delta / 2$. By substituting $N=0$ into Eq. (1), we obtain:

$$
\begin{aligned}
I(l) \propto & 2 \sqrt{I_{r e f} I_{o b j 1}} \times \exp \left[-\left(2 \sqrt{\ln 2} l / L_{c o h}\right)^{2}\right] \\
& \times \cos (k \times l)+2 \sqrt{I_{r e f} I_{o b j 2}} \\
& \times \exp \left[-\left(2 \sqrt{\ln 2}(l+\Delta) / L_{c o h}\right)^{2}\right] \\
& \times \cos (k \times(l+\Delta)) .
\end{aligned}
$$

From Eq. (3), when $l=0$ and $l=-\Delta$, we can observe the interference fringes by identical pulse trains. Eq. (3) means that the observed multiple pulse trains' interference fringes "de- generate" into white light interference fringes, the same as can be seen in white light interference (Figure 2).

Here, we briefly summarize the measuring principle of the white light interference method. White light interference is a well-established and powerful tool for non-contact 3D surface profilometry that permits rapid and accurate measurements without $2 \pi$ ambiguity. Because of the short coherence length of the white light source, a one-on-one relationship arises between the heights of the object surface and the peaks of the fringe-visibility curve. We can obtain the height of the surface corresponding to the location of the peak of the fringevisibility curve along the scanning axis.

The theoretical limits of white light interferometry have been fully described [21]-[24]. Before using the result of the uncertainties evaluation of the white light interference to evaluate the PRIEF method, we need to verify whether any change happened to the white light interference method by changing a white light source into an FOFC.

In a white light interferometer, there is no difference between a white light source and an FOFC. Basicly, we observe that identical light parts interfere with each other. With a white light source, the identical light parts are wave packets. With an FOFC source, the identical parts are pulse trains.

The PRIEF method and the white light interference method are the same for obtaining the distance from the peaks of the interference fringe, although the cause of the interference is different. The above-mentioned degeneration gives us a way to estimate the measuring error of the fraction part. In the PRIEF method, because the optical distance is relative large, atmospheric turbulence will affect the relative position between the peaks of an interference fringe, but the turbulence does not affect the measurement accuracy of the distance from the peaks. In other words, both methods have the same accuracy for determining the distance from the peaks of an interference fringes.

Therefore, by using the result of the uncertainties evaluation of the white light interference, we can conclude that the absolute measuring accuracy of the fraction part is nanometerorder, and the main influence factor is scan accuracy of the reference mirror.

Having discussed the measuring uncertainties used to determine the fraction part, we will next focus our attention on the 


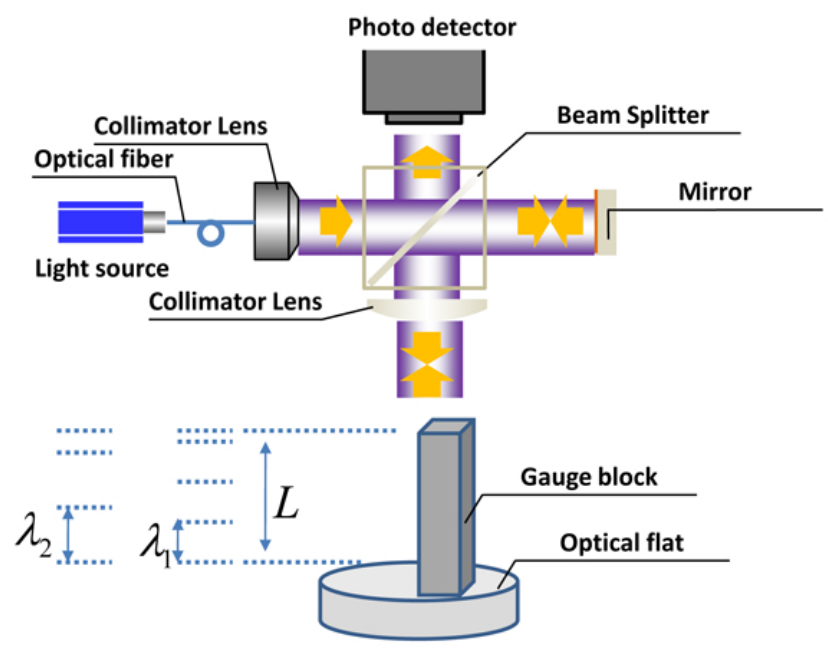

(a)

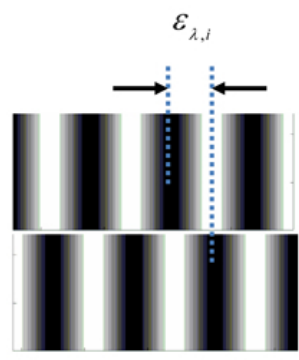

(b)

FIG. 3 Excess Fraction method. (a) Schematic diagram of optical setup. (b) Interference fringes.

uncertainties evaluation used to determine the integer part. Here we summarize the Excess Fraction method, which was proposed [19, 20] in the 1970s and widely applied [25]-[28]. An arbitrary and absolute distance $L$ with a rough knowledge of its range (namely, $L_{\text {rough }}$ in $\left[L_{\min }, L_{\max }\right]$ ) can be expressed by the following equation.

$$
\begin{aligned}
L & =\lambda_{1} \times\left(N_{\lambda, 1}+\varepsilon_{\lambda, 1}\right) / 2=\ldots=\lambda_{i} \times\left(N_{\lambda, i}+\varepsilon_{\lambda, i}\right) / 2 \\
& =\ldots=\lambda_{q} \times\left(N_{\lambda, q}+\varepsilon_{\lambda, q}\right) / 2,
\end{aligned}
$$

where the term $\lambda_{i}$ represents an already known and steady wavelength. $N_{\lambda, i}$ and $\varepsilon_{\lambda, i}$ are the integer part and the fraction part for wavelength $\lambda_{i}$, respectively. A schematic description of the optical set for the Excess Fraction method and a measuring fringe are shown in Figure 3. The fraction part $\varepsilon_{\lambda, i}$ can be measured from the displacement between the interference fringes. An integer part $N_{\lambda, i}$ can be decided by satisfying Eq. (5):

$$
\begin{aligned}
N_{\lambda, i}= & \min \left\{\sum_{i=1}^{q}\left[\frac{\lambda_{i} \times\left(N_{\lambda, i}+\varepsilon_{\lambda, i}\right)}{2}-L_{\text {rough }}\right]\right\} \\
& \& \lambda_{i} \times\left(N_{\lambda, i}+\varepsilon_{\lambda, i}\right) / 2 \in\left[L_{\min }, L_{\max }\right] .
\end{aligned}
$$

Then $L$ can be measured as:

$$
L_{\text {meas }}=\sum_{i=1}^{q}\left[\lambda_{i} \times\left(N_{\lambda, i}+\varepsilon_{\lambda, i}\right) / 2 q\right] .
$$

The combined standard uncertainty of the measured distance, $\sigma_{c}^{2}\left(L_{\text {meas }}\right)$ is given by: [26]

$$
\begin{aligned}
\sigma_{c}^{2}\left(L_{\text {meas }}\right)= & \sum_{i=1}^{q}\left[\left(\frac{\lambda_{i}}{2 q}\right)^{2} \sigma^{2}\left(N_{\lambda, i}\right)\right]+\sum_{i=1}^{q}\left[\left(\frac{\lambda_{i}}{2 q}\right)^{2} \sigma^{2}\left(\varepsilon_{\lambda, i}\right)\right] \\
& +\sum_{i=1}^{q}\left[\left(\frac{N_{\lambda, i}+\varepsilon_{\lambda, i}}{2 q}\right)^{2} \sigma^{2}\left(\lambda_{i}\right)\right]
\end{aligned}
$$

where $\sigma^{2}\left(\lambda_{i}\right)$ is the uncertainty of wavelength in air influences by stability of the wavelength of the light source for wavelength $\lambda_{i}$, and $\sigma^{2}\left(N_{\lambda, i}\right)$ and $\sigma^{2}\left(\varepsilon_{\lambda, i}\right)$ are the uncertainty for the integer part detection and fraction part detection, respectively.
The PRIEF method can be described and evaluated the same way.

An arbitrary and absolute distance $L$ can be expressed as:

$$
\begin{aligned}
L & =c \times T_{R, 1} \times\left(N_{T R, 1}+\varepsilon_{T R, 1}\right) / 2 \\
& =\ldots=c \times T_{R, i} \times\left(N_{T R, i}+\varepsilon_{T R, i}\right) / 2 \\
& =\ldots=c \times T_{R, q} \times\left(N_{T R, q}+\varepsilon_{T R, q}\right) / 2,
\end{aligned}
$$

where the term $T_{R, i}$ represents an already known and steady pulse repeat interval. $N_{T R, i}$ and $\varepsilon_{T R, i}$ are the integer part and the fraction part for pulse repeat interval $T_{R, i}$, respectively.

An integer part $N_{T R, i}$ can be decided using:

$$
\begin{aligned}
N_{T R, i}= & \min \left\{\sum_{i=1}^{q}\left[\frac{c \times T_{R, i} \times\left(N_{T R, i}+\varepsilon_{T R, i}\right)}{2}-L_{\text {rough }}\right]\right\} \\
& \& c \times T_{R, i} \times\left(N_{T R, i}+\varepsilon_{T R, i}\right) / 2 \in\left[L_{\min }, L_{\max }\right] .
\end{aligned}
$$

Then Lcan be measured as:

$$
L_{\text {meas }}=\sum_{i=1}^{q}\left[c \times T_{R, i} \times\left(N_{T R, i}+\varepsilon_{T R, i}\right) / 2 q\right] .
$$

The combined standard uncertainty of the measured distance, $\sigma_{c}^{2}\left(L_{\text {meas }}\right)$ is given as:

$$
\begin{aligned}
\sigma_{c}^{2}\left(L_{\text {meas }}\right)= & \sum_{i=1}^{q}\left[\left(\frac{c \times T_{R, i}}{2 q}\right)^{2} \sigma^{2}\left(N_{T R, i}\right)\right] \\
& +\sum_{i=1}^{q}\left[\left(\frac{c \times T_{R, i}}{2 q}\right)^{2} \sigma^{2}\left(\varepsilon_{T R, i}\right)\right] \\
& +\sum_{i=1}^{q}\left[\left(\frac{N_{T R, i}+\varepsilon_{T R, i}}{2 q}\right)^{2} \sigma^{2}\left(c \times T_{R, i}\right)\right]
\end{aligned}
$$

where $\sigma^{2}\left(c \times T_{R, i}\right)$ is the uncertainty of pulse repetition interval influences by stability of the pulse repetition interval of the FOFC source, and $\sigma^{2}\left(N_{T R, i}\right)$ and $\sigma^{2}\left(\varepsilon_{T R, i}\right)$ are uncertainties of integer part detection and fraction part detection, respectively.

Considering that $N_{T R, i}$ represents an integral part of interference orders for a long range, and that we can assume that the 
algorithm based on Eq. (9) has chosen the correct length solution, then $\sigma^{2}\left(N_{T R, i}\right)=0$.

We have discussed $\sigma^{2}\left(\varepsilon_{T R, i}\right)$ in the uncertainty evaluation of the integer part; let us turn our attention to $\sigma^{2}\left(c \times T_{R, i}\right)$. In a vacuum, the uncertainty of a pulse repeat interval is only reversely proportional to the achievable control accuracy of the repetition frequency. In air, the stability of a pulse repeat interval is smaller than $10^{-9}$, but the achievable accuracy of the refractive index of air is $10^{-8}$-order, usually. The uncertainty of a pulse repeat interval is factually restricted by the measurement uncertainty of the refractive index of air.

We think about measurement uncertainty by integrating the integer part and fraction part. In a comparison of $\sum_{i=1}^{q}\left[\left(N_{T R, i}+\varepsilon_{T R, i} / 2 q\right)^{2} \sigma^{2}\left(c \times T_{R, i}\right)\right]$ and $\sum_{i=1}^{q}\left[\left(c \times T_{R, i} / 2 q\right)^{2} \sigma^{2}\left(\varepsilon_{T R, i}\right)\right]$, the larger term will limit measurement accuracy. In vacuum, because the absolutely measurement uncertainty of $\sum_{i=1}^{q}\left[\left(c \times T_{R, i} / 2 q\right)^{2} \sigma^{2}\left(\varepsilon_{T R, i}\right)\right]$ is of nanometer-order, which is limit of absolutely accuracy of the PRIEF method. Because relative accuracy is proportional to the length range, we can expect to measure long distances with high accuracy. For example, measuring one kilometer with nanometer accuracy means that the relative accuracy is $10^{-12}$-order.

In air, because the achievable measuring accuracy of the refractive index of air is $10^{-8}$-order, the larger term normally $\sum_{i=1}^{q}\left[\left(N_{T R, i}+\varepsilon_{T R, i} / 2 q\right)^{2} \sigma^{2}\left(c \times T_{R, i}\right)\right]$, will limit measurement accuracy. For example, let's assume the measure target is one meter. That means the accuracy of the fraction part is nanometer-order, and the accuracy of the integer part is tens of nanometer-order. Therefore, the relative accuracy is $10^{-8}$ order and the absolute accuracy is tens of nanometers-order.

This theoretical expectation corresponds well to the results described in our previous report. [10] The achieved measuring accuracy of the refractive index of air was $10^{-6}$-order, which limited the relative accuracy.

\section{SUMMARY}

We have proposed a novel approach for the theoretical analysis of measurement limitations of the PRIEF method, by focusing on the analogy between the proposed method and the conventional length measurement method. The accuracy estimate was divided into two parts. Based on the analogy between the proposed method and the white light method, we conclude that the measurement accuracy of a fraction part can reach nanometer-order. Based on the analogy between the proposed method and the ordinary Excess Fraction method, we found the following two conclusions. First, in vacuum, long-distance measurement with high accuracy is promising. Second, in air, the estimation of the refractive index of the propagation medium is the bottleneck of the measurement accuracy of an integral part.
By comparing these two parameters of accuracy limit, we arrived at the following two conclusions. The first is that the minimum absolute accuracy achievable by the PRIEF method is nanometer-order, and it is limited by the scanning performance of the scanning device. The second finding is that the minimum relative accuracy achievable by the PRIEF method is $10^{-8}$-order, and is mainly affected by the measurement accuracy of the refractive index of the propagation medium. We expect that this theoretical analysis will lead to further use of the PRIEF method.

\section{ACKNOWLEDGEMENTS}

This research work was financially supported by a scientific research grant from the Mitutoyo Association for Science and Technology (R11 02), and by the "Development of System and Technology for Advanced Measurement and Analysis" Program at the Japan Science and Technology Agency (to H. M.).

\section{References}

[1] K. Minoshima, and H. Matsumoto, "High-accuracy measurement of 240-m distance in an optical tunnel by use of a compact femtosecond laser," Appl. Optics 39, 5512-5517 (2000).

[2] S. Yokoyama, T. Yokoyama, Y. Hagihara, T. Araki, and T. Yasui, "A distance meter using a terahertz intermode beat in an optical frequency comb," Opt. Express 17, 17324-17337 (2009).

[3] K. Minoshima, K. Arai, and H. Inaba, "High-accuracy self-correction of refractive index of air using two-color interferometry of optical frequency combs," Opt. Express 19, 26095-26105 (2011).

[4] H. Matsumoto, X. Wang, K. Takamasu, and T. Aoto, “Absolute Measurement of Baselines up to $403 \mathrm{~m}$ Using Heterodyne Temporal Coherence Interferometer with Optical Frequency Comb," Applied Physics Express 5, 046601 (2012).

[5] C. Narin, T. Satoru, T. Kiyoshi, and M. Hirokazu, "A new method for high-accuracy gauge block measurement using $2 \mathrm{CHz}$ repetition mode of a mode-locked fiber laser," Measurement Science and Technology 23, 054003 (2012).

[6] X. Wang, S. Takahashi, K. Takamasu, and H. Matsumoto, "Space position measurement using long-path heterodyne interferometer with optical frequency comb," Opt. Express 20, 2725-2732 (2012).

[7] D. Wei, K. Takamasu, and H. Matsumoto, "Pulse repetition intervalbased Excess Fraction method for length measurement directly linked to a femtosecond optical frequency comb," in Proceedings to FiO (Frontiers in Optics)/LS (Laser Science) 2011, FThU6 (Optical Society of America, 2011).

[8] D. Wei, K. Takamasu, and H. Matsumoto, "Pulse repetition intervalbased Excess Fraction method for an arbitrary and absolute distance measurement using a femtosecond optical frequency comb," in Proceedings to Frontiers in Ultrafast Optics: Biomedical, Scientific, and Industrial Applications XII, 82470K-82411 (SPIE, 2012).

[9] D. Wei, and H. Matsumoto, "Pulse repetition interval-based excess fraction (PRIEF) method and its measurement accuracy," in Proceedings to the European Optical Society (EOS) Annual Meeting 2012, TOM4_5791_001, (EOS, Aberdeen, 2012).

[10] D. Wei, S. Takahashi, K. Takamasu, and H. Matsumoto, “Time-offlight method using multiple pulse train interference as a time recorder," Opt. Express 19, 4881-4889 (2011). 
[11] D. Wei, S. Takahashi, K. Takamasu, and H. Matsumoto, "Theoretical Analysis of Length Measurement Using Interference of Multiple Pulse Trains of a Femtosecond Optical Frequency Comb," Japanese journal of applied physics 50, 022701 (2011).

[12] Y. Nakajima, H. Inaba, K. Hosaka, K. Minoshima, A. Onae, M. Yasuda, T. Kohno, et al., "A multi-branch, fiber-based frequency comb with millihertz-level relative linewidths using an intra-cavity electro-optic modulator," Opt. Express 18, 1667-1676 (2010).

[13] J. Ye, R. Jones, L. Chen, K. Holman, and D. Jones, "Applications of Femtosecond Laser Combto Nonlinear Molecular Spectroscopy Astrophysics, Clocks and Fundamental Constants," S. Karshenboim, E. Peik, eds., 275-295 (Springer, Berlin / Heidelberg, 2004).

[14] L. M. Nelson, J. Levine, and P. Hetzel, "Comparing primary frequency standards at NIST and PTB," in Proceedings to Frequency Control Symposium and Exhibition, 2000, 622-628 (IEEE, Kansas City, 2000).

[15] J. Ye, J.-L. Peng, R. J. Jones, K. W. Holman, J. L. Hall, D. J. Jones, S. A. Diddams, et al., "Delivery of high-stability optical and microwave frequency standards over an optical fiber network," J. Opt. Soc. Am. B 20, 1459-1467 (2003).

[16] E. Bengt, "The Refractive Index of Air," Metrologia 2, 71 (1966).

[17] K. P. Birch, and M. J. Downs, "An Updated Edlén Equation for the Refractive Index of Air," Metrologia 30, 155 (1993).

[18] P. E. Ciddor, "Refractive index of air: new equations for the visible and near infrared," Appl. Optics 35, 1566-1573 (1996).

[19] C. R. Tilford, "Analytical procedure for determining lengths from fractional fringes," Appl. Optics 16, 1857-1860 (1977).
[20] G. L. Bourdet, and A. G. Orszag, "Absolute distance measurements by $\mathrm{CO} 2$ laser multiwavelength interferometry," Appl. Optics 18, 225-227 (1979).

[21] M. Fleischer, R. Windecker, and H. J. Tiziani, "Theoretical Limits of Scanning White-Light Interferometry Signal Evaluation Algorithms," Appl. Optics 40, 2815-2820 (2001).

[22] M. E. Pawłowski, Y. Sakano, Y. Miyamoto, and M. Takeda, "Phasecrossing algorithm for white-light fringes analysis," Opt. Communications 260, 68-72 (2006).

[23] K. G. Larkin, "Efficient nonlinear algorithm for envelope detection in white light interferometry," J. Opt. Soc. Am. A 13, 832-843 (1996).

[24] A. Olszak, "Lateral Scanning White-Light Interferometer," Appl. Optics 39, 3906-3913 (2000).

[25] H. Matsumoto, "Length measurement using infrared twowavelength He-Xe laser interferometer," Review of Scientific Instruments 53, 641-643 (1982).

[26] J. E. Decker, and J. R. Pekelsky, "Uncertainty evaluation for the measurement of gauge blocks by optical interferometry," Metrologia 34, 479 (1997).

[27] H. Matsumoto, "Recent interferometric measurements using stabilized lasers," Precision Engineering 6, 87-94 (1984).

[28] K. Falaggis, D. P. Towers, and C. E. Towers, "Method of excess fractions with application to absolute distance metrology: theoretical analysis," Appl. Optics 50, 5484-5498 (2011). 